\title{
An Algorithm for the Construction of Optimal Methods for the Numerical Solution of Volterra Integral Equations of the First Kind
}

\author{
By C. J. Gladwin
}

\begin{abstract}
Optimal methods for the numerical solution of Volterra integral equations of the first kind are outlined in [3] and [4]. An explicit algorithm for the construction of such methods as well as tables of coefficients for methods with order less than or equal to eight are displayed here.
\end{abstract}

1. Introduction. Quadrature methods for the numerical solution of Volterra integral equations of the first kind are described by the ordered triple $Q(C, B, r)$, where:

(i) $C \in \mathbf{R}^{p+1}$ consists of the Newton-Cotes quadrature weights with step number $p$;

(ii) $B \in \mathbf{R}^{p+2}$ are coefficients of the polynomial

$$
\rho(z)=\sum_{i=0}^{p+1} b_{i} z^{p+1-i}
$$

(iii) $r$ is the order of the methods, $r \leqslant p+2$;

(iv) $p$ starting values are needed prior to the implementation of the method. See [3], [4] and [5] for details.

Here we shall only be concerned with giving a simple explicit algorithm to search for optimal methods $Q(C, B, r)$. A table of the coefficients, $B$, for $r \leqslant 8$ will also be given. The constraints on $B$ are two-fold. First, $r$ linear conditions must be satisfied to attain order $r$, and second, the roots of the polynomial $\rho(z)$ must lie in or on the unit circle with roots on the circle having multiplicity 1 . We have available $p+2-r \geqslant 0$ parameters to implement the slightly more restrictive form of the latter condition: All roots lie strictly inside the unit circle. Furthermore, we are only interested in methods satisfying the above constraints which have the least number of free parameters. It is known that $p+2-r=0$ for $p=0$ only; see [5] or [6]. We first give a short digression on some classical stability results for roots of polynomials.

Received October 13, 1982; revised February 27, 1985 and August 14, 1985.

1980 Mathematics Subject Classification. Primary 65R05; Secondary 45L10.

Key words and phrases. Quadrature rule methods, Volterra integral equations of the first kind, numerical stability. 
2. Schur and Hurwitz Polynomials. ([1] or [7].) All polynomials considered here will be assumed to be in the class $\pi_{n}$, the set of polynomials of exact degree $n$ with real coefficients and no roots at the origin.

Definition 1. The polynomial $a(z)=\sum_{i=0}^{n} a_{i} z^{i}$ is said to be a Schur polynomial if $a(z)=0$ implies $|z|<1$.

Definition 2. The polynomial $b(w)=\sum_{i=0}^{n} b_{i} w^{i}$ is said to be a Hurwitz polynomial if $b(w)=0$ implies $\operatorname{Re} w<0$.

We catalogue some relationships between these two classes of polynomials:

(i) $b(w)=(w-1)^{n} a((w+1) /(w-1)) \in \pi_{n}$,

(ii) $a(z)=((z-1) / 2)^{n} b((z+1) /(z-1)) \in \pi_{n}$.

(iii) The coefficients of $a(z)$ and $b(w)$ are related by the matrix relations:

$$
\begin{gathered}
B=\Gamma \cdot A, \\
A=2^{-n} \Gamma \cdot B
\end{gathered}
$$

with $A=\left(a_{0}, a_{1}, \ldots, a_{n}\right), B=\left(b_{0}, b_{1}, \ldots, b_{n}\right)$, and $\Gamma=\left(\gamma_{i, j}\right)$ the matrix of order $n+1$ generated by the recursive scheme:

$$
\begin{aligned}
& \gamma_{i-1, j-1}=\gamma_{i-1, j}-\gamma_{i, j-1}-\gamma_{i, j}, \\
& \gamma_{n, i}=1, \gamma_{j, n}=\left(\begin{array}{l}
n \\
j
\end{array}\right),
\end{aligned} \quad i, j=1(1) n .
$$

(iv) The polynomial $a(z)$ is a Schur polynomial if and only if $b(w)$ is a Hurwitz polynomial.

(v) The polynomial $a(z)$ is a Schur polynomial if and only if:

(a) $\left|a_{0}\right|<\left|a_{n}\right|$

(b) $a_{1}(z)=\sum_{i=0}^{n-1}\left(a_{n} a_{i+1}-a_{0} a_{n-(i+1)}\right) z^{i}$ is a Schur polynomial.

In later applications, it will be necessary to normalize the coefficients of $a_{1}(z)$, leading coefficient unity at each step, due to the rapid growth of the coefficients of $a_{1}(z)$.

We shall use the notation $D^{i}\{p(z)\}$ to denote the $i$ th derivative of $p(z)$ with respect to $z$.

(vi) If $p(z)$ is a Schur (or Hurwitz) polynomial then $D^{i}\{p(z)\}, i=0(1) n$, are also Schur (or Hurwitz) polynomials. The contra-positive of this statement will be of use in the algorithm.

3. The Algorithm. We return to the consideration of $\rho(z)$, defined in (1.1), and also the transformed version

$$
\tau(w)=(w-1)^{p+1} \rho\left(\frac{w+1}{w-1}\right) .
$$

The order conditions imposed on $\rho(z)$ are given by the linear system

$$
\sum_{i=0}^{p+1} i^{j} b_{i}=\frac{1}{j+1}, \quad j=0(1) r-1,
$$

with the convention $0^{0}=1$.

It was shown in [3] that

$$
\tau(w)=\sum_{i=r}^{p+1} q_{i} 2^{i}(w-1)^{p+1-i}+(w-1)^{p+1} \sum_{i=0}^{r-1} b_{p+1-i}\left(\frac{w+1}{w-1}\right)^{i},
$$


where $q_{i}$ are linear in $b_{i}, i=0(1) p+1-r$. This enables us to use the linear system

$$
D^{i}\left\{\tau\left(w_{0}\right)\right\}=0, \quad i=0(1) p+1-r,
$$

to determine the remaining parameters so that $\tau(w)$ (or $\rho(z)$ ) has as many equal real roots as possible; see also [3]. Here, $w_{0}$ is selected from the at most $r-1$ real roots of $D^{p+2-r}\{\tau(w)\}$ so that $\tau(w)$ is a Hurwitz polynomial, if possible.

Unfortunately, the resulting linear relations (3.4) for the $b_{i}, i=0(1) p+1-r$, become quite unwieldy because of the nature of the $q_{i}$. In this note, we show how one can solve for $\tau_{i}, i=0(1) p+1$, and then transform back using (2.1b).

THEOREM 1. Let $\rho_{0}(z)$ be as in (1.1) with coefficients satisfying (3.2) and $b_{i}=0$, $i=0(1) p+1-r$. Further, let

$$
\tau_{0}(w)=(w-1)^{r-1} \rho_{0}((w+1) /(w-1)) \text { and } c(w)=(w-1)^{p+2-r} \tau_{0}(w) .
$$

Then

(i) $D^{p+2-r}\{\tau(w)\}=D^{p+2-r}\{c(w)\}$,

(ii) $\tau(w)=\sum_{i=0}^{p+1-r} \tau_{i} w^{i}+\sum_{i=p+2-r}^{p+1} c_{i} w^{i}$,

where $c_{i}$ are the coefficients of $c(w)$ and $\tau_{i}$ are to be determined.

Proof. For (i), we have from (3.3)

$$
\begin{aligned}
D^{p+2-r}\{\tau(w)\} & =D^{p+2-r}\left\{\sum_{i=0}^{r-1} b_{p+1-i}(w+1)^{i}(w-1)^{p+1-i}\right\} \\
= & D^{p+2-r}\left\{(w-1)^{p+2-r}(w-1)^{r-1} \sum_{i=0}^{r-1} b_{p+1-i}(w+1)^{i}(w-1)^{-i}\right\} \\
= & D^{p+2-r}\{c(w)\} .
\end{aligned}
$$

(ii) follows by integrating $D^{p+2-r}\{\tau(w)\} p+2-r$ times so that $\tau_{i}, i=0(1) p+$ $1-r$, are simply the integration constants which may be determined by the conditions (3.4).

Remark. For ease of notation, subscripts " $p$ " and/or " $r$ " will be addended to the polynomials $\rho_{0}, \tau_{0}$, and $c$ only when necessary. (See also Theorem 2.)

We now summarize the algorithm.

(i) For each $p=1,2,3, \ldots$, consider $r=p+1(-1) 2$.

(ii) Solve (3.2) with $b_{i}=0, i=0(1) p+1-r$, to obtain $\rho_{0}(z)$.

(iii) Generate $\tau_{0}(w)$ using $(2.1 \mathrm{a})$.

(iv) Generate $c(w)=(w-1)^{p+2-r} \tau_{0}(w)=\sum_{i=0}^{p+1} c_{i} w^{i}$.

(v) Generate $D^{i}\{c(w)\}, i=0(1) p+2-r$. Only the $r$ coefficients $c_{i}, i=p+$ $2-r(1) p+1$, are needed.

(vi) Solve for the $r-1$ roots of $D^{p+2-r}\{c(w)\}$. Note that if any of these roots have nonnegative real parts, go to (v) and take the next $r$; see also Section 4 .

(vii) Otherwise, for a real root $w_{0}$, solve the linear system

$$
\sum_{j=i}^{p+1-r}\left\{i !\left(\begin{array}{l}
j \\
i
\end{array}\right) w_{0}^{j-i}\right\} \tau_{j}=-\sum_{j=p+2-r}^{p+1} i !\left(\begin{array}{l}
j \\
i
\end{array}\right) c_{j} w_{0}^{j-i}, \quad i=0(1) p+1-r .
$$

The matrix of coefficients of (3.5) is an upper triangular Wronskian with determinant $\prod_{i=1}^{p+1-r} i$ !. 
(viii) Generate $B \in \mathbf{R}^{p+2}$, applying (2.1b) to the vector $\left(\tau_{0}, \ldots, \tau_{p+1-r}, c_{p+2-r}, \ldots, c_{p+1}\right)$, and test if the resulting $B$ is a vector of Schur coefficients using (v) of Section 2.

(ix) If it is, proceed to (i) and increment $p$. Otherwise, try the next real root of $D^{p+2-r}\{c(w)\}$ and return to (vii).

(x) If none is available, proceed to (v) and try the next $r \geqslant 2$.

Remark. Any method with $r=2$ and $p>0$ is stable (Schur). We show that $\tau(w)$ is a Hurwitz polynomial, i.e.,

$$
c_{p+1}=\sum_{i=0}^{p+1} b_{p+1-i} \gamma_{p+1, i}=\sum_{i=0}^{p+1} b_{i}=1
$$

and

$$
c_{p}=\sum_{i=0}^{p+1} b_{p+1-i} \gamma_{p, i}=\sum_{i=0}^{p+1} b_{p+1-i}[2 i-(p+1)]=p,
$$

using the first two equations of (3.2). Hence, $D^{p+2-r}\{\tau(w)\}$ has a single real root at $w_{0}=-p /(p+1)$, and thus $\tau(w)$ has a root of multiplicity $p+1$ at this $w_{0}$. Of course, this method is only optimal in the case $p=1$ in the sense that the number of free parameters is a minimum.

4. Alternative Method of Calculation of $\rho_{0}(z)$. The calculation of the coefficients of $\rho_{0}(z)$ in step (ii) involves inversion of a Vandermonde matrix which can be quite ill-conditioned for large $r$ and $p(r=9, p=13)$. Here we derive some recursive relations for computing $\rho_{0}(z)$ for various $r$ and $p$. Furthermore, it will be shown that only $\rho_{0}(z)$ for $r=p+1$ need be calculated; see also (vi) of the algorithm.

We denote

$$
\rho_{0}(z)=\sum_{i=1}^{r} s_{r, p, i} z^{r-i}
$$

where

$$
s_{r, p, i}=\frac{(-1)^{r-i}}{r-1 !}\left(\begin{array}{c}
r-1 \\
i-1
\end{array}\right) \int_{0}^{1} \prod_{\substack{j=1 \\
j \neq i}}^{r}[x-(p+1-r+j)] d x
$$

[8, p. 152]; i.e., $\rho_{0}(z)$ is as in (1.1) with $r$-order conditions (3.2) as well as $b_{i}=0$, $i=0(1) p+1-r$, imposed. The following lemma summarizes some recursive relations needed.

LEMMA 1. We have

(i)

$$
s_{r, p, i}=(-1)^{r-i}\left(\begin{array}{c}
r-1 \\
i-1
\end{array}\right) s_{r, p, r}+s_{r-1, p-1, i}, \quad i=r(-1) 1, s_{r-1, p-1, r}=0
$$

(ii)

$$
\sum_{i=0}^{r-1} \frac{(-1)^{i}}{i+1} s_{r-i, p, r-i}=(-1)^{r+1}, \quad r=p+1, s_{1, p, 1}=1
$$

$$
s_{r, p, i}=(-1)^{i-1}\left(\begin{array}{c}
r-1 \\
i-1
\end{array}\right) s_{r, p, 1}+s_{r-1, p, i-1}, \quad i=1(1) r, s_{r-1, p, 0}=0 .
$$


Proof. For (i), we have

$$
s_{r, p, i}=\frac{(-1)^{r-i}}{r-1 !}\left(\begin{array}{c}
r-1 \\
i-1
\end{array}\right) \int_{0}^{1} \frac{[x-(p+2-r)] \cdots[x-(p+1)]}{[x-(p+1-r+i)]} d x .
$$

Using $x-(p+1)=x-(p+1-r+i)-(r-i)$ and splitting up the integrand, we obtain (i). (iii) is obtained in a similar fashion using $x-(p+2-r)=x-$ $(p+1-r+i)+i-1$. Finally, for (ii), we have

$$
s_{r, p, r}=\frac{1}{r-1 !} \int_{0}^{1}[x-(p+2-r)] \cdots[x-p] d x .
$$

Making the change of variable $u=x-(p+2-r)$ and defining

$$
G(t)=\sum_{r=1}^{\infty} s_{r, p, r} t^{r-1}
$$

we get the generating function

$$
G(t)=\frac{(1+t)^{-(p+2-r)} t}{\log (1+t)}
$$

Using the expansion for $\log (1+t)$ and equating equal powers of $t$ (for $r=p+1$ ), we obtain (ii).

Equations (i) and (ii) are used to compute $\rho_{0}(z)$ for $r=p+1$ and each $p=1(1) \infty$. Equation (iii) will be needed to justify the need of calculating $\rho_{0}(z)$ for $r=p+1$ only.

THEOREM 2. Let $\rho_{0, r}(z), \tau_{0, r}(w)$, and $c_{r}(w)$ be defined as in Theorem 1 . Then

$$
c_{r}(w)-c_{r-1}(w)=s_{r, p, 1} 2^{r-1}(w-1)^{p+2-r} .
$$

Proof. A simple calculation with the aid of (iii) in Lemma 1 yields

$$
\rho_{0, r}(z)-\rho_{0, r-1}(z)=s_{r, p, 1}(z-1)^{r-1} .
$$

Note that $p$ is a fixed subscript in the recursive relation (iii). Replace $z$ by $(w+1) /(w-1)$ and multiply by $(w-1)^{r-1}(w-1)^{p+2-r}=(w-1)^{p+1}$. Thus we obtain (4.3).

Remark. Since $c(w) \in \pi_{p+1}$ for every $r$ (with fixed $p$ ), the coefficients $c_{i}$, $i=p+2-r(1) p+1$, must be invariant with $r$. See also (ii) of Theorem 1 . Thus $\rho_{0}(z)$ need only be calculated for $r=p+1$. See also (v) and (vi) of the algorithm.

5. Table of Coefficients. We first present a table of the optimal $r$ for each $p$. This is given for the coefficients of $\rho(z)$ as well as for $\rho_{0}(z)$; i.e., for the crude choice $b_{i}=0, i=0(1) p+1-r$.

The coefficients of $\rho_{0}(z)$ are the Adams-Bashforth weights $(p \leqslant 5)$; compare also [2, p. 194]. The methods derived here are of use for $6 \leqslant p \leqslant 20$. For completeness we present the coefficients of $\rho(z)$ for $p \leqslant 10$ in Table 2 . Tables and programs for arbitrary $p$ and $r$ are available from the author. 
TABLE 1

Optimal $r ; p+2-r$ is minimum

\begin{tabular}{|c|c|c|}
\hline$p$ & $\rho(z)$ & $\rho_{0}(z)$ \\
\hline 1 & 2 & 2 \\
2 & 3 & 3 \\
3 & 3 & 4 \\
4 & 4 & 5 \\
5 & 5 & 6 \\
\hline 6 & 5 & 5 \\
7 & 6 & 5 \\
8 & 7 & 5 \\
9 & 7 & 5 \\
10 & 8 & 5 \\
11 & 8 & 5 \\
12 & 9 & 5 \\
13 & 10 & 5 \\
14 & 10 & 5 \\
15 & 11 & 5 \\
16 & 11 & 5 \\
17 & 11 & 5 \\
18 & 11 & 5 \\
19 & 11 & 5 \\
20 & 12 & 5 \\
\hline
\end{tabular}

6. Conclusions. The coefficients in Table 2 were prepared on a Honeywell Sigma 9 computer at the Communications Research Centre in Ottawa, Canada. Double precision was used, i.e., a 64-bit word. While the coefficients of $D^{p+2-r}\{\tau(w)\}$ are rational, hence the roots necessarily algebraic, the coefficients of $\tau(w)$ or $\rho(z)$ may become algebraic when repeating an irrational $w_{0}$. See $p=2$, for example. If $w_{0}$ is complex, $\tau(w)$ could be made to have a repeated complex factor $\left(w-w_{0}\right)\left(w-\bar{w}_{0}\right)$ $=w^{2}-2 \operatorname{Re}\left(w_{0}\right) w+\left|w_{0}\right|^{2}$, since we are really only interested in making $\tau(w)$ have as many roots as possible of equal magnitude; see also [3]. No attempts at this have been made here, as it would only be practical in a case where all the roots of $D^{p+2-r}\{\tau(w)\}$ have negative real parts and all the real roots lead to unstable methods. This does occur in the $(p, r)$ pairs: $(17,12),(18,12),(19,13),(19,12)$, $(20,14),(20,13)$ so that, perhaps, the orders for $17 \leqslant p \leqslant 20$ may be increased, by consideration of complex roots with negative real parts. See also Table 1.

Methods with less than optimal order (more free parameters than necessary) are not entirely "useless". It is sometimes useful to choose a polynomial $\rho(z)$ so that the weights of the overall quadrature rule, $Q(C, B, r)$, have the same sign. This will not be pursued here.

We close with a couple of conjectures concerning properties of $D^{p+2-r}\{\tau(w)\}$.

(i) All roots are simple.

(ii) There exists at most one root $w_{0}$ which makes $\tau(w)$ a Hurwitz polynomial. The basis of this latter conjecture is that the parameter subspace, $\mathbf{R}^{p+2-r}$, may be partitioned into at most $p+2$ sets where $\tau(w)$ has $k$ roots with negative real parts, $0 \leqslant k \leqslant p+1$, and a given $w_{0}$ determines (uniquely) a point in one of these sets. 
TABLE 2

Coefficients of $\rho(z)$ for optimal $(p, r)$ pairs. The coefficients correspond to ascending powers of $z$ (reading down a column)

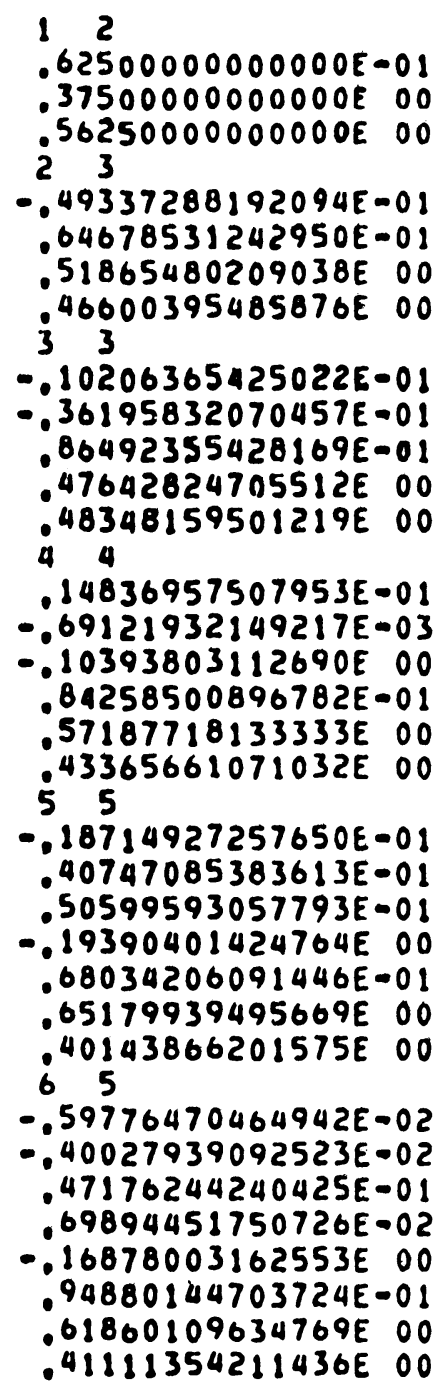

$$
\begin{aligned}
& 76 \\
& .92163405049008 E-02 \\
& \therefore 14546809675587 E-01 \\
& \because 48304895337707 E-01 \\
& .97947306939138 E-01 \\
& .72442509783543 E-01 \\
& \text {-.26845326473361E 0O } \\
& .70903231800597 E-01 \\
& .68397907230593 E 00 \\
& .38781850832280 E \text { OO } \\
& 87 \\
& -.129451574362535 .01 \\
& .43708651498921 E-01 \\
& \text {. } 22125052690577 E-02 \\
& . .16620573410212 E 00 \\
& .15292714699083500 \\
& .19610793531520 E \text { OO } \\
& .38565080617132 E 00 \\
& .56506829998068 E-01 \\
& .74345535496711 E 00 \\
& .36988327367051 E 00 \\
& 97 \\
& \text {-.49091448505026E-02 } \\
& .5227336580596 \text { BE-02 } \\
& .36113819972249 E-01 \\
& \text {-.47656074893641E-01 } \\
& -.10003946902624 E 00 \\
& .17585056164945 E 00 \\
& .10722157092790 E 00 \\
& \therefore 34934604089284 E 00 \\
& .85779797591605 E-01 \\
& .71530363345109 E 00 \\
& .37645400949034 E 00 \\
& 100 \\
& .77531170545616 E-02 \\
& -.23039576731772 E-01 \\
& \text {-.21648723845614E-01 } \\
& .13000342387131 E 00 \\
& \text {-43085358541170E-01 } \\
& \because 27790370100636 E 00 \\
& .26254546035601 E 00 \\
& .24470889259012 E 00 \\
& . .47277597813738 E \text { OO } \\
& .64252391412316 E-01 \\
& .76720259863094 E \text { OO } \\
& .36198745434704 E 00
\end{aligned}
$$

7. Acknowledgment. The author would like to thank the Communications Research Centre (Department of Communications, Ottawa) for providing the necessary computing facilities used in the preparation of this paper. 
42 Empire Street

Bridgewater, Nova Scotia

Canada B4V 2L4

1. R. J. Duffin, “Algorithms for classical stablity problems," SIAM Rev., v. 11, 1969, pp. 196-213.

2. P. HenricI, Discrete Variable Methods in Ordinary Differential Equations, Wiley, New York, 1962.

3. C. J. Gladwin, "On optimal integration methods for Volterra integral equations of the first kind," Math. Comp., v. 39, 1982, pp. 511-518.

4. C. J. Gladwin, "Quadrature rule methods for Volterra integral equations of the first kind," Math. Comp., v. 33, 1979, pp. 705-716.

5. C. J. Gladwin, Numerical Solution of Volterra Integral Equations of the First Kind, Ph. D. Thesis, Dalhousie University, Halifax, N. S., 1975.

6. C. J. Gladwin \& R. JeLtsCH, "Stability of quadrature rules for first kind Volterra integral equations," BIT, v. 14, 1974, pp. 144-151.

7. M. Marden, Geometry of Polynomials, 2nd ed., Math. Surveys, No. 3, Amer. Math. Soc., Providence, R. I., 1966.

8. A. Ralston, A First Course in Numerical Analysis, MacGraw-Hill, New York, 1965. 